\title{
Praxisanleitung in der Radiologie - ein aktueller Stand
}

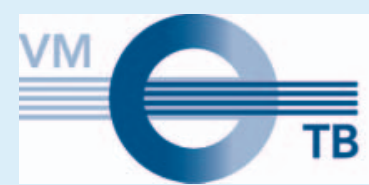

VEREINIGUNG MEDIZINISCH-TECHNISCHER BERUFE

in der Deutschen Röntgengesellschaft e.V.

Zu Beginn des Jahres 2023 erreichen wir in der Ausbildung von Radiologie-Technologinnen und -Technologen einen neuen Meilenstein, weil das MTA-Gesetz aus dem Jahr 1993 und die zugehörige MTA-Ausbildungs- und Prüfungsverordnung aus den Folgejahr dann (endlich) der Vergangenheit angehören wird. Allerdings ist mit dieser $\mathrm{Er}$ leichterung die große Sorge verbunden, nicht allen Forderungen in der Ausbildung unseres Nachwuchses gerecht zu werden.

Einer dieser Punkte betrifft die Praxisanleitung in der praktischen Ausbildung. In der novellierten Gesetzesfassung wird von einer praxisanleitenden Person gesprochen, die „die Auszubildenden an die praktischen und berufsspezifischen Tätigkeiten in der medizinischen Technologie ... [heranführt]“ und „den Lernprozess während der praktischen Ausbildung“ begleitet ( $\$ 20$, MTBG).

Praxisanleitung ist in der Ausbildung im Gesundheitswesen nichts Neues, denn insbesondere in den Pflegeberufen ist diese schon seit vielen Jahren etabliert. Der Einsatz praxisanleitender Personen sowie die Anforderungen, die an sie gestellt werden, sind in den jeweiligen Ausbildungs- und Prüfungsverordnungen grob umrissen und beinhalten in der künftigen MTR-Ausbildung Folgendes (siehe $\S 8$, MTAPrV):

a) Die Erlaubnis zum Führen der Berufsbezeichnung nach dem MTA-Gesetz oder nach dem MT-Berufe-Gesetz.

b) Eine mindestens einjährige Berufserfahrung.

c) Eine absolvierte berufspädagogische Zusatzqualifikation mit einem Umfang von mindestens 300 Stunden.

d) Jährliche berufspädagogische Fortbildungen mit einem Umfang von mindestens 24 Stunden.
Die Anforderungen zu a) und b) sind eindeutig und bedürfen sicherlich neben dem Hinweis, dass folgerichtig Medizinischen Fachangestellten nicht als praxisanleitende Person eingesetzt werden dürfen, keiner weiteren Erläuterungen.

Bezüglich der geforderten berufspädagogischen Zusatzqualifikation (c) gibt es keine weiteren formulierten Anforderungen. Hier müssen die jeweilig zuständigen Aufsichtsbehörden der Bundesländer unter Umständen aktiv werden. Da es für die MT-BerufeAusbildung bisher keine expliziten Regelungen gibt, lohnt sich ein kleiner Seitenblick auf die Ausbildung in den Pflegeberufen. Beispielsweise heißt es dazu in einem Erlass des Ministeriums für Arbeit, Gesundheit und Soziales des Landes Nordrhein-Westfalen (MAGS) bezüglich der Praxisanleitung nach dem Pflegeberufegesetz: „Für Nordrhein-Westfalen gilt weiterhin, dass Weiterbildungsstätten zur Durchführung der Weiterbildung Praxisanleitung keiner gesonderten staatlichen Anerkennung bedürfen“ (Erlass vom 25. Februar 2020).

Bezogen auf die Inhalte dieser Weiterbildung gibt es ebenfalls keinerlei konkrete Vorgaben in der künftigen MT-Ausbildungs- und Prüfungsverordnung. Aus dem oben erwähnten Erlass lässt sich implizit jedoch ableiten, dass vorwiegend berufspädagogische sowie berufsfachliche und berufspolitische Inhalte zu vermitteln sind.

Diese angeführten Punkte, wie auch die Aussagen einiger Behördenvertreter, lassen den Schluss zu, dass Personen mit einer abgeschlossenen Berufsausbildung nach dem „alten“ MTA-Gesetz von 1993, die in der Vergangenheit eine berufspädagogische Weiterbildung absolviert haben zukünftig als ausgebildete praxisanleitende Personen eingesetzt werden dürfen, wenn sie ab
2023 kontinuierlich die geforderten berufspädagogischen Fortbildungen nachweisen. Zudem gilt nach $\S 8$, Nr. 2 (MTAPrV) für Personen, die am 31.12.2022 als praxisanleitende Person eingesetzt und den zuständigen Behörden gemeldet sind, Bestandsschutz; auch für sie gilt dann die kontinuierliche Fortbildungsverpflichtung.

Die Inhalte dieser oben erwähnten berufspädagogischen Fortbildungen (d) werden leider in den Verordnungen auch nicht näher erläutert, sondern müssen letztendlich auf Ebene der Bundesländer geregelt werden. Möglicherweise hilft der bereits erwähnte Erlass des nordrhein-westfälischen Gesundheitsministeriums auch hierbei weiter, wenn dort folgende Kriterien für besagte Fortbildungen aufgeführt werden:

- Die Fortbildungszeit von 24 Stunden kann in der Regel in maximal 4 Veranstaltungen aufgeteilt werden.

- Der zeitliche Überhang der 24 Stunden Fortbildungszeit eines Jahres kann nicht auf kommende Jahre übertragen werden.

- Die Fortbildungen können berufspädagogische (mind. $50 \%$ ), berufsfachliche und berufspolitische Inhalte haben.

Die in dieser Zusammenfassung wiedergegebenen Inhalte aus dem Erlass des nordrhein-westfälischen MAGS sind sicherlich nicht in Gänze auf die Regelungen in anderen Bundesländern übertragbar. Sie spiegeln jedoch in etwa die zukünftigen Rahmenbedingungen der Praxisanleitung in der MT-Berufe-Ausbildung wider. Konkrete Absprachen sind seitens der Ausbildungseinrichtungen mit den zuständigen Behörden des jeweiligen Bundeslandes zu klären.

Die Einführung einer möglichst einheitlich organisierten Praxisanleitung soll die praktische MTR-Ausbildung inhaltlich und strukturell bereichern. Für die Umsetzung dieser gesetzlichen Vorgaben sind die gegebenen infrastrukturellen Gegebenheiten der Ausbildungsstätte wie auch Regelungen des jeweiligen Bundeslandes zu berücksichtigen. 